Vol. 3, No. 1 | January - June 2019

\title{
Aspects Based Opinion Mining for Teacher and Course Evaluation
}

Sarang Shaikh ${ }^{1}$, Sher Muhammad Doudpotta ${ }^{1}$

\begin{abstract}
:
Teacher and course evaluation by students at the end of each term is an important task in almost every academic institution worldwide. It helps in assessing faculty performance and suitability of the course in any academic program. The data collected from evaluation comprises of two parts, Likert Scale and open-ended feedback. Computationally, the Likert Scale form can be handled easily as it is numerical in nature but to handle open-ended feedback is a challenging task. Presently, in most of the organizations it is processed manually, which contains many problems like it is error-prone, tedious and full of human biases. The objective of this study is to solve these problems, using two-step rule-based strategy from Machine Learning and Natural Language Processing (NLP) techniques. The first step is to extract overall topic of the feedback text using supervised machine learning followed by exploitation of NLP rules to find out specific aspect and related opinion word about which the feedback is given along with orientation of the opinion either positive, negative or neutral. Using, this two-step strategy combining with NLP, machine learning techniques and data from past seven years of real feedback at a public sector university in Pakistan, we are able to achieve a recall and precision of $83.89 \%$ and $84 \%$ on topic identification i.e. to classify a feedback in teacher and course category. The system is able to extract different aspects of teacher and course with a precision of $83 \%$ and recall of $80 \%$, whereas overall sentiment classification accuracy is $90 \%$. To the best of our knowledge, this is the first rule-based approach for such problem with quite satisfactory results.
\end{abstract}

Keywords: Opinion Mining, Natural Language Processing (NLP), Text Mining \Teacher's / Course Evaluation.

\section{Introduction}

Student feedback/ evaluation about teacher and course at the end of every term is an important integral part of any academic organization. The impact of this evaluation can be understood from its outcomes which most of the times highlights teacher's positive areas and identifies areas of improvement. Also, this evaluation helps to find out the impact of course regarding specific degree program. The evaluation is also helpful for the annual progress of faculty members [1]. Mainly, this evaluation involves student's feedback regarding different aspects of teacher and course. For Example: teacher's behavior, methodology, teaching pace, course contents, etc. Student feedback helps in most of the areas where there is a need of any improvement. Ultimately, this evaluation process using student's feedback assists top-level management to make

\footnotetext{
${ }^{1}$ Department of Computer Science, Sukkur IBA University Sukkur, Pakistan Corresponding Email: sarang.msse17@iba-suk.edu.pk
}

decisions for further continuation of those faculty members or courses. [3].

The student's feedback collected during evaluation process consists of mainly two types, Quantitative Feedback and Qualitative Feedback. The quantitative feedback is taken from a student using Likert Scale whereas the qualitative feedback is in form of open-ended textual comments regarding teacher and course [1]. Processing quantitative feedback is an easier task as performance can be represented in terms of percentages which are easy to comprehend. On the other hand, the textual feedback is hard to represent in numbers, consequently, it is mostly processed manually, which means human involvement. The task of processing text is tedious, especially when student population is high, just imagine 5000 students in a university, more than 200 faculty members and 100s of courses, the 
amount of data to process simply requires an army of readers, even then, it would be an error-prone and full of human biases. Ironically, a university with mentioned population is considered a small university, medium and large universities population is many folds of this definition.

Considering this major issue, the research community is working towards the machine-based evaluation of these openended comments. This machine-based evaluation of unstructured textual data lies under the category of natural language processing (NLP), text mining and more specifically opinion mining / sentiment analysis [2]. In recent years, many attempts have been made under the umbrella of opinion mining including variety of approaches using machine learning classification models and lexical approaches [5]. Liu et al. in [6] and Moraes et al. in [12] have defined opinion mining problem as general document classification problem which considers single review as single subjective document classified as either negative, positive or neutral.

This paper proposes two-step strategy based on natural language processing and machine learning techniques. In the first step, we use supervised machine learning to identify the topic of feedback, either teacher or course, followed by employing natural language rules to extract specific aspect of the teacher or course which is being discussed in the text. Finally, classification of the text in positive, negative and neutral classes. Furthermore, using this approach a standard corpus has been developed for teacher/course evaluation domain using data from past seven years from a public sector university in Pakistan as a training model. The corpus consists of important aspects in this domain for which most of the time students discuss their possible opinion words with desired sentiment classes. The proposed solution works for automatic sentiment classification for opinions collected during evaluation task at universities. Rest of the paper is organized as: section 2 discusses related work, section 3 explains methodology, section 4 explains results and section 5 explains conclusion \& the future work.

\section{Related Works}

There are many recent attempts to automate sentiment analysis in general and students' opinions in particular. This section, describes opinion mining, its basic concepts followed by opinion mining application on teacher / course evaluation using students' feedback. [3]

\section{a. Opinion Mining}

Esuli et al. in [4] define opinion mining as a sub discipline of the two major disciplines --- information retrieval and computational linguistics, which doesn't explain topic of text but expression / meaning of text. Mishra et al. in [5] define opinion mining is a technique which takes some of data mining techniques to identify speaker's / writer's attitude towards particular topic and overall sentiment orientation of particular block of text. Liu et al. in [6] define term opinion as a quintuple in Eq. 1. For a text, which contains subjective opinion for different aspects the quintuple is defined by:

$$
e_{i}, a_{i, j}, o o_{i j k l}, h_{k}, t_{l}
$$

where

$\mathrm{e}_{i}$ is entity name

$\mathrm{a}_{i j}$ is the attribute of entity

$\mathrm{oo}_{i j k l}$ is sentiment orientation of attribute

$\mathrm{h}_{k}$ is opinion holder

$t_{l}$ is time when opinion was expressed.

Considering this definition of opinion, Cambria et al. in [7] define opinion mining as a process to apply different tasks by discovering all quintuples from opinion texts.

\section{b. Existing Approaches}

Below are some major approaches which literature suggests regarding opinion mining as this research area has recently got a lot of attention.

\section{i. Document Sentiment Classification:}

This approach can also be termed as machine learning based opinion mining. The important discussion under this approach is that it considers each opinion, piece of text as single document so according to this approach every document represents subjectivity regarding only single entity. i-e. it considers a single review about a product or service. The major drawback of this approach is that it works for general aspect of feedback text. As this is machine learning based approach so it is divided into two types:

- Supervised Document Sentiment Classification

- Unsupervised Document Sentiment Classification The authors of [8] applied this approach for student's feedback text by using different classifiers including Naive Bayes, Maximum Entropy and Support Vector Machine (SVM). The author got maximum accuracy of $94 \%$ for their dataset on SVM 


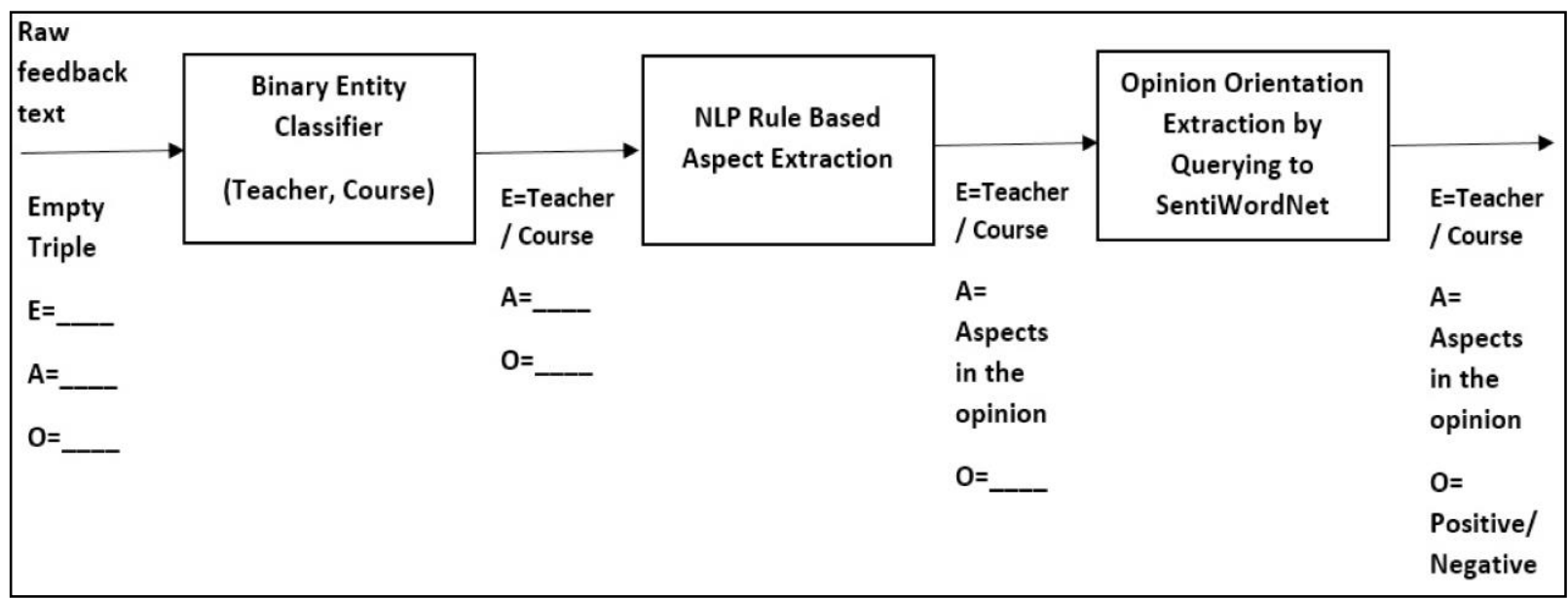

Figure 1:Proposed System Overview

classifier. On the other hand, Turney et al. in [9] applied this approach on movie reviews dataset and got overall accuracy of $84 \%$.

\section{ii. Opinion Lexical Approach:}

Taboada et al. in [10] define this approach as to calculate sentiment orientation of document using polarity scores of words or phrases in the document using some pre-built dictionaries or corpus. Lexical approach consists of two methodologies. [3]

- Dictionary Based Approach

- Corpus Based Approach

Liu et al. in [6] explained dictionary-based approach to create small opinion words seed list, then use an online dictionary like WORDNET [11] to query limited opinion words for finding their synonyms and antonyms and the process continues until no new word is found from dictionary. [10]

Liu et al. in [6], explain corpus-based approach works same as dictionary-based approach defined above but major difference is involvement of some sentiment consistency inference rules to avoid domain inconsistency. Particularly, if two opinion words are combined with each other using conjunction "and", if first opinion word is positive oriented so inference rules suggests second opinion word to be positive oriented too. This approach supports most of these inference rules. [10]

\section{iii. Aspect Based Sentiment Analysis Approach:}

Pontiki et al. in [14] define this approach as finding sentiments about specific entities and their aspects for which feedback is provided. The previous literature suggested two major approaches but major drawback in those approaches is they provide overall sentiment of specific text but this is not necessarily that if feedback is overall positive then it cannot have any negative aspect. The feedback is always combination of different positive and negative opinions regarding different aspects or attributes of entities [6]. For example, students give positive opinion regarding course content but give negative opinion regarding teacher methodology. Mubarok et al. in [15] applied this approach using Naive Bayes Classifier on restaurant domain customer reviews data set and got accuracy of $78.12 \%$.

\section{iv. Spam Feedback Detection:}

Opinions/reviews available over the web for different products or items have gained major attention from different users before buying any item. This practice is widely used to identify major aspects of products or items. These reviews mainly explain reputation of product. More the positive reviews, more the product is likely to be accepted otherwise rejected. Liu. et al. in [6] has explained spam feedback as false or fake reviews which are used to mislead readers to increase or decrease chances of item selection. Jindal \& Liu. et al. in [21], [22] had proposed the idea of detecting fake opinion, first time. They define spam feedback detection using below methods: 
- $\quad$ Spam Detection Based on Supervised Learning

- Spam Detection Based on Abnormal Behaviors Spam detection based on supervised learning is a classificationbased approach which contains two classes, spam and nonspam. Duplicate reviews or near-to-duplicate reviews are classified as spam reviews, whereas the rest were classified as non-spam reviews. Jindal. et al. in [24] have defined another technique which identifies unusual reviewer behavior using unexpected rule discovery.

\section{v. Existing Approaches for Teachers Evaluation:}

Rajput Q. et al. in [3] used corpus-based approach and got $91.2 \%$ accuracy on real data of their university. The work is limited only on sentiment orientation classification, whereas extraction of different aspects of course and teacher have not been considered in this work. Altrabsheh et al. in [8] used machine learning based approach using Naive Bayes, SVM classifier and got accuracy of 94\%. Chee. in [26] used sentiment analysis on (SMS) texts in teacher evaluation. The authors have proposed models-based approach which leads to the base model, corrected model and sentiment model. Altrabsheh. et al. in [27] have applied sentiment analysis in education domain for teacher's evaluation. Inspite of using data from stored files, they have used social networks like Facebook, twitter to get data and perform sentiment analysis. There are many other authors who have worked on sentiment analysis for teacher's evaluation domain but to analyze feedback in this domain using aspect-based approach is an active research problem and same is the problem addressed in this paper.

\section{System Overview}

Fig. 1 shows the abstract model of the proposed system. The proposed system accepts raw opinionated text at the input to extract entity, aspect and opinion orientation. The first step based on supervised learning is a binary classifier that takes the input text and classifies it in either teacher or course entities. Once the entity has been extracted, the next step is to use natural language processing rules to learn relationship between words and different part of speeches to extract aspect of the entity. For example, behavior, contents knowledge, experience and assessment are aspect for teacher entity, whereas pace, market acceptability, suitability in curriculum are some aspect for the course entity. Finally, we use different part of speech words to query SentiWordNet to extract orientation of an opinion. Suppose, following raw text is input,

"Teaching methodology of this teacher is excellent, he teaches in a way that lindent difficult topics become easy"

For this text, following triple would be generated,

$(\mathrm{E}, \mathrm{A}, \mathrm{O})=($ Teacher, Teaching Methodology, Positive $)$

Similarly, for following text,

"I don't know why they are still teaching this course, job market no more lindent require graduate to know GWBasic"

Here, the triple would be,

$(\mathrm{E}, \mathrm{A}, \mathrm{O})=($ Course, Market Acceptability, Negative $)$

In next few sections, we describe working of each of these components of the proposed system.

\subsection{Binary Entity Classifier}

We have used major text pre-processing techniques (See Section 3.1.1) and applied text features engineering (See Section 3.1.2) combined with classification algorithm (See Section 3.1.3) to classify text as whether it belongs to teacher or course entity. We have used 10,000 manually annotated feedback for teacher / course category. The annotation has been done by the domain experts. We divided feedback into training and test instances with $70 \% 30 \%$ ratio. Fig. 2 shows samples taken from training data.

\begin{tabular}{|ll|}
\hline For teacher & good teacher \\
For teacher & awesome teacher and we love to be taught by him again \\
For teacher & always my favorite. \\
For teacher & This teacher is quite well who try that students learn something helps us generate our analytical skills \\
For teacher & never seen that like teacher of finance in my academic life. he is expert in his field. \\
For Course & a helpful course for beginners. \\
For Course & informative course \\
For Course & good course. \\
For Course & good teacher and good teaching, methodology. \\
For Course & Course rating is normal \\
\hline
\end{tabular}

Figure 2: Manual Labelled Input Raw Text for 
It has been observed that usually noun, adjective and verbs differentiate well between entities of two different domains, namely teacher and course. All other words present in the raw text are considered stop words and removed during preprocessing step, this reduces vocabulary of the text and also improves efficiency. Algorithm 1 shows main steps involved in entity extraction.

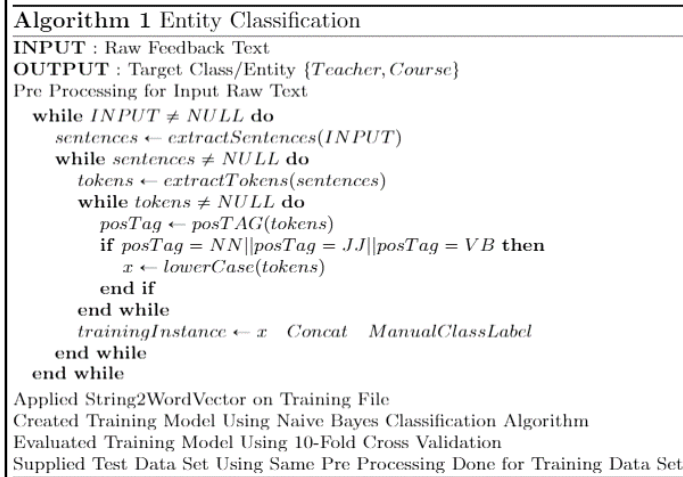

\subsubsection{Text Pre-Processing}

As given in Algorithm 1, we have performed 04 pre-processing steps. [16]

We have used default Apache OpenNLP models to perform preprocessing tasks. Finally, the extracted words for each sentence are stored as training instance with their desired manual class label (see Fig. 3). Any classification task expects features in numerical format, therefore, we need to convert our training data, which is in text format, to numbers.

\subsubsection{Feature Extraction}

We use String2WordVector to create features/attributes from text to be used for classification. String2WordVector uses concepts from language modeling called Term FrequencyInverse Document Frequency (TF*IDF). In [17] Zhang et al. has defined TF*IDF in detail. (See Eq.2)

For a term $i$ in a document $j$, the weight $W_{i, j}$ of term $i$ in document $j$ is given by:

$$
W_{i, j}=t f_{i, j} * \log \left(\frac{N}{d f i}\right)
$$

where

- $\mathrm{tf}_{\mathrm{i}, \mathrm{j}}$ is the number of occurrences of $\mathrm{i}$ in document $\mathrm{j}$.

- $\mathrm{df}_{\mathrm{i}}$ is the number of documents containing the term $\mathrm{i}$.

- $\mathrm{N}$ is the total number of documents in the corpus.

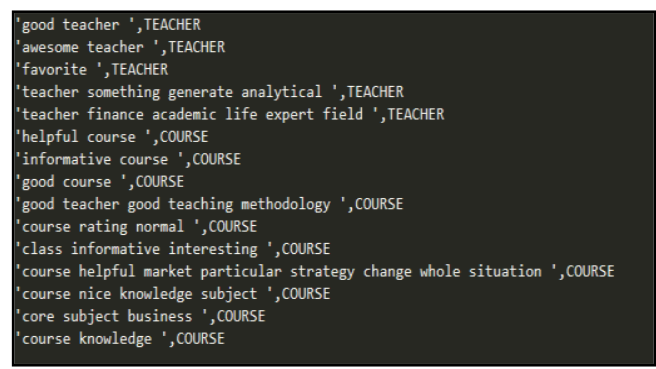

Figure 3: Sample Training Instances File

\subsubsection{Naive Bayes Multinomial Classification}

$\mathrm{Xu}$. et al. in [18] has explained this classification algorithm as one variation of Naive Bayes classifier, best for multinomial distributed data which in case of text classification is best suited. As compared to other algorithms this is faster and accurate specifically for text classification. The author has discussed complete mathematics and procedure of this classification algorithm as defined in Fig.4

For each document $m \in\{1, \ldots, M\}$ :

(a) Draw a class $c_{m}$ from $\operatorname{Multinomial}(\vec{\vartheta})$;

(b) For each word $n \in\left\{1, \ldots, N_{m}\right\}$ in document $m$ : i. Draw a word $w_{m, n}$ from Multinomial $\left(\vec{\varphi}_{c_{m}}\right)$.

Figure.4: The mathematical form of Naïve Bayes Multinomial Classifier

As explained earlier, the outcome of this classification task is entity extraction, either teacher or course, from the raw input text. This means, we have filled the first term of our triple that expects entity, aspect and opinion orientation. The next section focuses on aspect extraction.

\subsection{NLP Rule Based Aspect Extraction}

Once the feedback text is classified into its desired entity whether teacher or course then it is used to extract desired

\begin{tabular}{|l|}
\hline Algorithm 2 Semantic Graph Dependencies Extraction \\
\hline INPUT : Classified Input Sentence \\
OUTPUT : Semantic Graph Dependencies of Input Sentence \\
while INPUT $\neq$ NULLL do \\
step1_(Output $\leftarrow$ applyCoreNLPAnnotators(INPUT) \\
step2_Output $\leftarrow$ generateGrammaticalStructures(step1_Output) \\
step3_(Output $\leftarrow$ createSemanticGraphTypedDependencies(step2_(output) \\
semanticGraphOutput $\leftarrow$ step3_(Output \\
end while
\end{tabular}

aspects (e-g: teacher's behavior, methodology, attitude, approach or course's contents, quality, etc.), their relevant 
opinions (e-g: good, best, pathetic, worst) and semantic orientation of those opinions (e-g: positive, negative or neutral). To achieve above, we have used Stanford CoreNLP API in JAVA. Algorithm 2. explains these steps.

\subsection{Semantic Graph Dependencies}

The common annotators provided by CoreNLP which we are using are tokenize, ssplit, pos, lemma, ner, parse, mention, dcoref. Manning. et al. in [19], the developers of these annotators have discussed all annotations in detail with purpose of each annotator. The next step is to create parse tree from annotations to understand word associations and relations. See Fig.5 for further explanation.

Once the parse tree for desired input is generated using parse annotator, the Grammatical Structure stores dependency relations between the nodes in a tree. It actually defines in detail hierarchy for input text using grammatical dependencies in terms intermediate step before going to actual step which is Step\\#3.

The output from step2 in form of tree and grammatical structures gives support to generate type dependencies of desired sentence using CoreNLP Semantic Graph Dependencies. It is used for generating syntactic dependencies of words in given sentence. These dependencies are based on sentence annotations, providing alot of support to traverse sentence for different grammatical formats for understanding its structure and writing style. see Fig. 6 for sample output generated by CoreNLP Semantic Graph Dependencies.

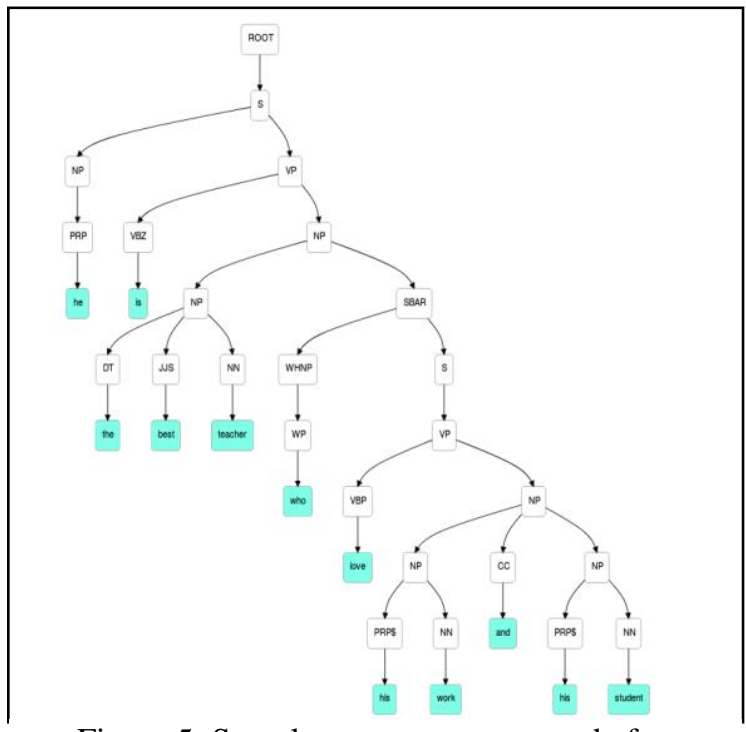

Figure 5: Sample parse tree generated after annotations

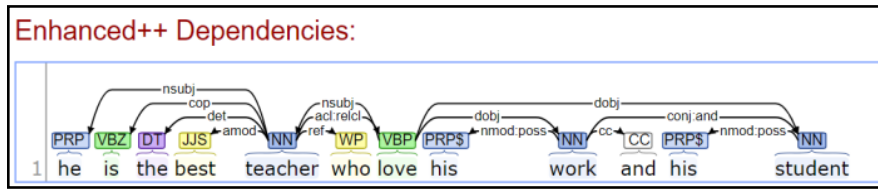

Figure 6: Semantic Graph Dependency Graph View

\subsection{Aspects, Opinion Extraction}

As discussed previously in section 3.3, we have seen output of semantic graphs dependencies. These dependencies are used to extract aspect, their opinions using rule-based approaches developed against different grammatical structure patterns. Below section will explain different rules developed for extraction on top of typed dependencies extracted using semantic graphic dependencies. See Fig. 7 for rules developed for extraction of aspects and their desired opinions from

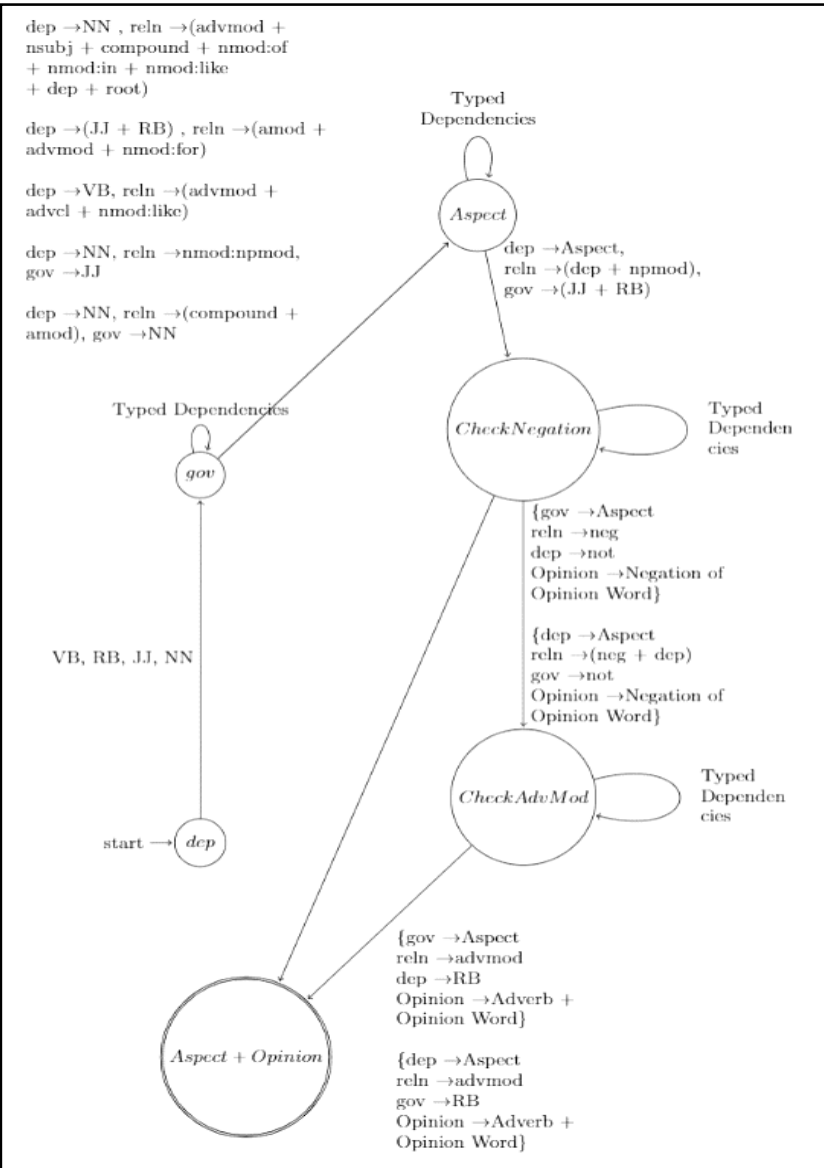

Figure 7. Aspect Opinion Extraction Rules 
feedback text. This figure represents extraction flow in form of state diagram.

Algorithm 3 explains flow of extraction and calculating semantic orientation using SentiWordNet [20].

Table 5 explains some of major supplied test opinions with details of desired extracted component. The semantic orientation calculation of opinion words is based on Eq. 3

$$
\begin{gathered}
\sum_{n=0}^{\text {count (opinionTokens) }} \text { calculatePolarity }(n) \rightarrow \\
\text { SentiWordNetCorpus }
\end{gathered}
$$

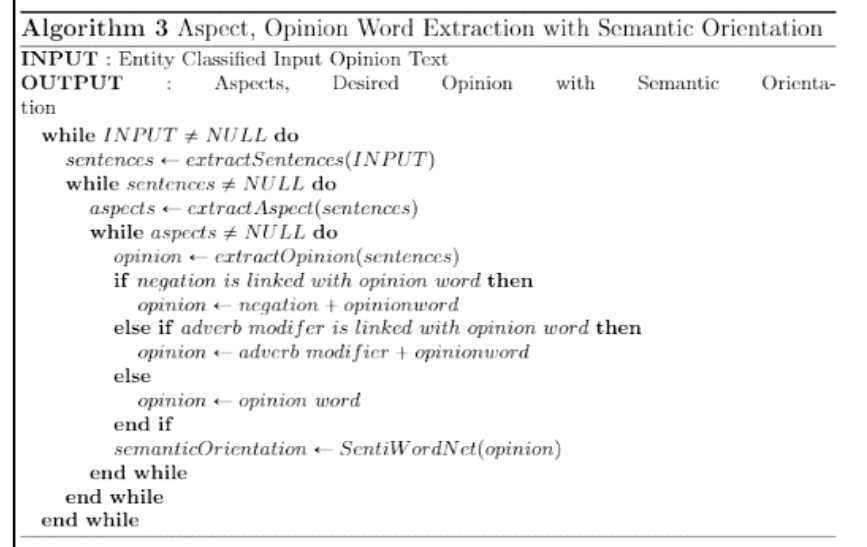

\subsection{Data Bank}

We have used $\$ 10,000 \$$ labelled student feedback texts collected during teacher's / course evaluation task at the end of academic semester at Sukkur IBA University Pakistan.

The collected texts are labelled by domain experts into three classes positive, negative and neutral, manually. These texts are used as initial data to run test cases and to evaluate proposed strategy for aspect-based opinion mining. Moreover, the feedback is in the form of raw text and stored into Microsoft Excel File with their desired label. see Table 1.

If we focus on third and fourth comment in Table I, it is labelled as negative opinion by domain expert as the domain expert knows meanings of opinions discussed against course as well as teacher and also knows relations between words. This is a major motivation for us going towards aspect-based opinion mining so that machine can also identify and separate different aspects and also can find out relations of words with each other. Our proposed model is able to identify such confusing comments correctly.

Table I: Raw Feedback Text Sample Used

\begin{tabular}{|l|l|}
\hline Feedback Text & $\begin{array}{l}\text { Manual } \\
\text { Labelled } \\
\text { Class }\end{array}$ \\
\hline course was very interesting and very good & positive \\
\hline course was useful & positive \\
\hline $\begin{array}{l}\text { subject is interesting but due to teacher it } \\
\text { become the boring one for me }\end{array}$ & negative \\
\hline $\begin{array}{l}\text { well instructor but he should try to improve } \\
\text { his teaching skills }\end{array}$ & negative \\
\hline Subject is ok & neutral \\
\hline it should be more updated & neutral \\
\hline
\end{tabular}

\section{Results}

In this section, we show the results of feedback text entity classifier, aspect and opinion extraction rules as well as semantic orientation of extracted opinions.

\subsection{Entity Classifier Results}

Table II \& III shows our experimental results on both entities (e$\mathrm{g}$ : teacher, course) by using 10-fold cross validation for total 7,675 instances. We present our results in precision and recall as defined in Eq.4 \& Eq.5.

$$
\begin{aligned}
& \text { Precision }=\frac{\text { No.of correctly classified entity }}{\text { Total classified entities }} \times 100 \\
& \text { Recall }=\frac{\text { No.of correctly classified entity }}{\text { No.of actual entities }} \times 100
\end{aligned}
$$

The average precision and recall results for teacher and course entity class were $84 \%$ and $83 \%$ respectively

Table II: Classification Results for TEACHER Entity Class

\begin{tabular}{|c|l|l|l|l|}
\hline Actual Instances & $\begin{array}{l}\text { Machine Classified } \\
\text { Instances }\end{array}$ & $\begin{array}{l}\text { Correctly Classified } \\
\text { Instances }\end{array}$ & Precision & Recall \\
\hline 4,324 & 4,720 & 3,904 & $82.8 \%$ & $90.3 \%$ \\
\hline
\end{tabular}


Table III: Classification Results for COURSE Entity Class

\begin{tabular}{|c|l|l|l|l|}
\hline Actual Instances & $\begin{array}{l}\text { Machine Classified } \\
\text { Instances }\end{array}$ & $\begin{array}{l}\text { Correctly Classified } \\
\text { Instances }\end{array}$ & Precision & Recall \\
\hline 3,351 & 2,955 & 2,535 & $85.5 \%$ & $75.6 \%$ \\
\hline
\end{tabular}

Table IV: Aspect, Opinion Word Extraction with Semantic Orientation (Precision, Recall) Results

\begin{tabular}{|l|l|l|l|l|l|}
\hline & $\begin{array}{l}\text { Actual Aspects } \\
\text { with Opinion }\end{array}$ & $\begin{array}{l}\text { System Extracted } \\
\text { Aspects with Opinion }\end{array}$ & $\begin{array}{l}\text { Correct System Extracted } \\
\text { Aspects with Opinion }\end{array}$ & Precision & Recall \\
\hline TEACHER & 25 & 24 & 21 & $87.5 \%$ & $84 \%$ \\
\hline COURSE & 20 & 19 & 15 & $79 \%$ & $75 \%$ \\
\hline OVERALL & 45 & 43 & 36 & $83 \%$ & $80 \%$ \\
\hline
\end{tabular}

Table V: Sample Results for Aspect, Opinion Word Extraction with Semantic Orientation Results

\begin{tabular}{|l|l|l|l|}
\hline Input Test Sentence & Extracted Aspects & $\begin{array}{l}\text { Extracted Aspects with } \\
\text { Opinions }\end{array}$ & $\begin{array}{l}\text { Opinion } \\
\text { Semantic } \\
\text { Orientation }\end{array}$ \\
\hline he is my favorite teacher & teacher & teacher (favorite) & POSITIVE \\
\hline He is a very good teacher and the course is awesome & teacher, course & $\begin{array}{l}\text { teacher (very good) } \\
\text { course(awesome) }\end{array}$ & $\begin{array}{l}\text { POSITIVE, } \\
\text { POSITIVE }\end{array}$ \\
\hline $\begin{array}{l}\text { he is one of teacher which teach us in very nice and } \\
\text { effective way }\end{array}$ & teacher's way & teacher's way (nice, effective) & POSITIVE \\
\hline $\begin{array}{l}\text { It is a challenging course as we are studying it for the } \\
\text { first time but all the things are very basic. }\end{array}$ & course & course (challenging) & NEGATIVE \\
\hline good behavior & teacher's behavior & teacher's behavior (good) & POSITIVE \\
\hline $\begin{array}{l}\text { A great teacher having vast experience and } \\
\text { knowledge. He always follows student centered } \\
\text { pproach }\end{array}$ & $\begin{array}{l}\text { teacher, teacher's } \\
\text { experience, teacher's } \\
\text { approach }\end{array}$ & $\begin{array}{l}\text { teacher (great), teacher's } \\
\text { experience (vast), teacher's } \\
\text { approach (centered) }\end{array}$ & $\begin{array}{l}\text { POSITIVE, } \\
\text { POSITIVE, } \\
\text { POSITIVE }\end{array}$ \\
\hline He is not a very good teacher & teacher & NEGATIVE \\
\hline $\begin{array}{l}\text { great teacher. tremendous efforts he did for each } \\
\text { student. }\end{array}$ & $\begin{array}{l}\text { teacher, teacher's } \\
\text { efforts }\end{array}$ & $\begin{array}{l}\text { teacher (great), teacher's } \\
\text { efforts (tremendous) }\end{array}$ & $\begin{array}{l}\text { POSITIVE, } \\
\text { POSITIVE }\end{array}$ \\
\hline course is bit difficult. & course & course (difficult) & NEGATIVE \\
\hline this subject helps me a lot to learn the new things & course's things & course's things (new) & POSITIVE \\
\hline $\begin{array}{l}\text { the nature of course was conceptual and very } \\
\text { informative }\end{array}$ & course's nature & $\begin{array}{l}\text { course's nature (very } \\
\text { informative) }\end{array}$ & POSITIVE \\
\hline worst learning Objective & course's learning & course's learning (worst) & NEGATIVE \\
\hline $\begin{array}{l}\text { good teacher and good teaching methodology } \\
\text { teacher, teacher's } \\
\text { methodology }\end{array}$ & $\begin{array}{l}\text { teacher, teacher's } \\
\text { methodology (good) }\end{array}$ & course's skills (analytical) & POSITIVE \\
\hline $\begin{array}{l}\text { course has a lot of things to learn from it. it has } \\
\text { nhanced my analytical skills }\end{array}$ & course's skills & NaL \\
\hline \hline
\end{tabular}




\subsection{Aspects, Opinion Extraction Rules Results}

We evaluated real time student's feedback to extract aspects and opinion words based on rules developed using CoreNLP Semantic Graph Dependencies in Fig 7. Some feedback was selected to test these rules for extraction, based on different grammatical patterns which convey one or more opinions regarding some aspects of teacher entity or course entity. Table IV summarizes our results of aspect extraction. Table V shows sample results of aspect extraction and opinion orientation from our experiments. We achieved an overall accuracy of $90 \%$ on opinion orientation extraction on our test dataset.

\section{Limitations \& Future Work}

The major limitations of this system are 1) Input of such new feedback text structures of which the system is unaware and cannot correctly extract the desired aspects as well as opinion words. 2) Usage of SentiWordNet to query opinion words for polarity assignment, there is a chance of occuring such opinion words which do not exists in SentiWordNet. 3) Occurance of roman urdu language-based students' feedback. 4) Usage of wrong English words spellings in feedback text. We will try to approach these limitations in future work of this research study.

\section{Conclusion}

Teachers and course evaluation are an important task in academia to analyze or assess performance of teachers and relevance of course in any academic program. Most of the time, the written feedback text do not give overall opinion for desired entity (i-e teacher or course), but it discusses different aspects of those entities.

In this research, we proposed a syntactic rule-based system for automatic aspects extraction and its polarity analysis from student's feedback given for a teacher or course. The proposed solution consisted of three steps, 1) Entity classification of feedback text whether it is for a teacher or course. 2) The classified text is given to developed rule-based system to analyze and extract aspects as well as opinion words from text using predefined rules. 3) Once the desired aspects are extracted, the system calculates semantic orientation of the opinion words using SentiWordNet for overall assignment of polarity score to all opinion words regarding aspects in a text. We got quite satisfactory results with overall precision of $83.89 \%$ and $84 \%$ on entity classification. The system extracted different aspects of teacher and course with a precision of $83 \%$ and recall of $80 \%$, whereas overall sentiment classification accuracy is $90 \%$. The results are very much satisfying as compared to research studies done in the past for the same domain as discussed in section existing approaches of related works portion of this paper.

\section{REFERENCES}

[1] Swapna Gottipati, Venky Shankararaman, and Sandy Gan. "A conceptual framework for analyzing students' feedback". In:Frontiers in Education Conference (FIE) . IEEE. 2017, pp. $1-8$.

[2] Quratulain Rajput, Sajjad Haider, and Sayeed Ghani. "Lexicon-Based Sentiment Analysis of Teachers' Evaluation". In: Applied Computational
Intelligence and Soft Computing 2016 (2016), p. 1. [3] Shiliang Sun, Chen Luo, and Junyu Chen. "A review of natural language processing techniques for opinion mining systems”. In: Information Fusion 36 (2017), pp. 10-25.

[4] Brojo Kishore Mishra and Abhaya Kumar Sahoo. "Evaluation of Faculty Performance in Education System Using Classification Technique in Opinion Mining Based on GPU'. In: Computational Intelligence in Data MiningVolume 2. Springer, 2016, pp. 109-119.

[5] Bing Liu and Lei Zhang. "A survey of opinion mining and sentiment analysis". In: Mining text data. Springer, 2012, pp. 415-463.

[6] Rodrigo Moraes, Jo ao Francisco Valiati, and Wilson P. Gavi ao Neto. "Document-level sentiment classification: An empirical comparison between SVM and ANN". In:Expert Systems with Applications 40.2 (Feb. 2013), pp. 621-633. doi: 10.1016/j.eswa.2012.07.059. url:

https://doi.org/10.1016/j.eswa.2012.07.059

[7] Andrea Esuli and Fabrizio Sebastiani. "SentiWordNet: a high-coverage lexical resource for opinion mining". In: Evaluation 17 (2007), pp. 1-26.

[8] Erik Cambria et al. "New avenues in opinion mining and sentiment analysis". In:IEEE Intelligent Systems 28.2 (2013), pp. $15-21$.

[9] Nabeela Altrabsheh, Mihaela Cocea, and Sanaz Fallahkhair. "Learning sentiment from students' feedback for real-time interventions in classrooms". In: Adaptive and Intelligent Systems. Springer, 2014, pp. 40-49.

[10] Peter D Turney. "Thumbs up or thumbs down?: semantic orientation applied to unsupervised classification of reviews". In:Proceedings of the

40th annual meeting on association for computational linguistics Association for Computational Linguistics. 2002, pp. $417-424$.

[11] Maite Taboada et al. "Lexicon-based methods for sentiment analysis". In: Computational linguistics 37.2 (2011), pp. 267-307.

[12] George A Miller. "WordNet: a lexical database for English". In: Communications of the ACM 38.11 (1995), pp. 39-41.

[13] Maria Pontiki et al. "SemEval-2016 task 5: Aspect based sentiment analysis". In: Proceedings of the 10th international workshop on semantic evaluation (SemEval2016) . 2016, pp. 19-30. 
[14] Mohamad Syahrul Mubarok, Adiwijaya, and Muhammad Dwi Aldhi. "Aspect-based sentiment analysis to review products using Naive Bayes".

In: AIP Conference Proceedings Vol. 1867. 1. AIP

Publishing. 2017, p. 020060.

[15] Xiaowen Ding, Bing Liu, and Philip S Yu. "A holistic lexicon-based approach to opinion mining". In: Proceedings of the 2008 international conference on web search and data mining . ACM. 2008, pp. 231-240.

[16] Nitin Jindal and Bing Liu. "Review spam detection". In: Proceedings of the 16th international conference on World Wide Web - WWW '07. ACM Press, 2007. doi:

10.1145/1242572.1242759. url:

https://doi.org/10.1145/1242572.1242759.

[17] Nitin Jindal, Bing Liu, and Ee-Peng Lim. "Finding unusual review patterns using unexpected rules". In:

Proceedings of the 19th ACM international conference on Information and knowledge management . ACM. 2010, pp. 1549-1552.

[18] Chee Kian Leong, Yew Haur Lee, and Wai Keong Mak. "Mining sentiments in SMS texts for teaching evaluation". In: Expert Systems with Applications 39.3 (2012), pp. 2584-2589.

[19] Nabeela Altrabsheh, M Gaber, and Mihaela Cocea. "SA-E: sentiment analysis for education". In:

International Conference on Intelligent Decision

Technologies. Vol. 255. 2013, pp. 353-362.

[20] Beatrice Santorini. "Part-of-speech tagging guidelines for the Penn Tree- bank Project (3rd revision)". In: Technical Reports (CIS)

(1990), p. 570.

[21] Wen Zhang, Taketoshi Yoshida, and Xijin Tang. "A comparative study of TF* IDF, LSI and multi words for text classification". In: Expert Systems with Applications 38.3 (2011), pp. 2758-2765.

[22] Shuo Xu, Yan Li, and Zheng Wang. "Bayesian Multinomial Navve Bayes Classifier to Text Classification". In: Advanced multimedia and ubiqui-

tous engineering. Springer, 2017, pp. 347-352.

[23] Kalina Bontcheva and Zhu Jingbo. "Proceedings of 52nd Annual Meeting of the Association for Computational Linguistics: System Demonstrations". In: Proceedings of 52nd Annual Meeting of the Association for Computational Linguistics: System Demonstrations. 2014.

[24] Stefano Baccianella, Andrea Esuli, and Fabrizio Sebastiani. "Sentiwordnet 3.0: an enhanced lexical resource for sentiment analysis and opinion mining." In: Lrec . Vol. 10. 2010. 2010, pp. 2200-220 
